\title{
Microbiota-sensitive epigenetic signature predicts inflammation in Crohn's disease
}

\author{
Daniel Kelly, ${ }^{1,2}$ Michael Kotliar, ${ }^{3}$ Vivienne Woo, ${ }^{1}$ Sajjeev Jagannathan, ${ }^{3}$ Jordan Whitt, ${ }^{1}$ \\ Jessica Moncivaiz, ${ }^{1}$ Bruce J. Aronow, ${ }^{4}$ Marla C. Dubinsky, ${ }^{5}$ Jeffrey S. Hyams, ${ }^{6}$ James F. Markowitz, ${ }^{7}$ \\ Robert N. Baldassano, ${ }^{8}$ Michael C. Stephens, ${ }^{9}$ Thomas D. Walters, ${ }^{10}$ Subra Kugathasan, ${ }^{11}$ \\ Yael Haberman, ${ }^{2,12}$ Nambirajan Sundaram, ${ }^{13}$ Michael J. Rosen, ${ }^{2}$ Michael Helmrath, ${ }^{13}$ Rebekah Karns, ${ }^{2}$ \\ Artem Barski, ${ }^{3}$ Lee A. Denson, ${ }^{2}$ and Theresa Alenghat ${ }^{1}$ \\ 'Division of Immunobiology, Center for Inflammation and Tolerance, 2Division of Gastroenterology, Hepatology, and \\ Nutrition, ${ }^{3}$ Divisions of Allergy and Immunology and Human Genetics, and ${ }^{4}$ Division of Biomedical Informatics, Cincinnati \\ Children's Hospital Medical Center (CCHMC) and Department of Pediatrics, University of Cincinnati College of Medicine, \\ Cincinnati, Ohio, USA. ${ }^{5}$ Department of Pediatrics, Mount Sinai Hospital, New York, New York, USA. ${ }^{6}$ Division of Digestive \\ Diseases, Hepatology, and Nutrition, Connecticut Children's Medical Center, Hartford, Connecticut, USA. 'Department \\ of Pediatrics, Northwell Health, New York, New York, USA. ${ }^{8}$ Department of Pediatrics, University of Pennsylvania, \\ Philadelphia, Pennsylvania, USA. 'Department of Pediatric Gastroenterology, Mayo Clinic, Rochester, Minnesota, USA. \\ ${ }^{10}$ Division of Pediatric Gastroenterology, Hepatology, and Nutrition, Department of Pediatrics, The Hospital for Sick \\ Children, University of Toronto, Toronto, Canada. "'Division of Pediatric Gastroenterology, Emory University School of \\ Medicine, Atlanta, Georgia, USA. ${ }^{12}$ Sheba Medical Center, Tel Hashomer, affiliated with the Tel-Aviv University, Israel. \\ ${ }^{13}$ Division of Pediatric General and Thoracic Surgery, CCHMC and Department of Pediatrics, University of Cincinnati College \\ of Medicine, Cincinnati, Ohio, USA.
}

Altered response to the intestinal microbiota strongly associates with inflammatory bowel disease (IBD); however, how commensal microbial cues are integrated by the host during the pathogenesis of IBD is not understood. Epigenetics represents a potential mechanism that could enable intestinal microbes to modulate transcriptional output during the development of IBD. Here, we reveal a histone methylation signature of intestinal epithelial cells isolated from the terminal ilea of newly diagnosed pediatric IBD patients. Genes characterized by significant alterations in histone H3-lysine 4 trimethylation (H3K4me3) showed differential enrichment in pathways involving immunoregulation, cell survival and signaling, and metabolism. Interestingly, a large subset of these genes was epigenetically regulated by microbiota in mice and several microbiota-sensitive epigenetic targets demonstrated altered expression in IBD patients. Remarkably though, a substantial proportion of these genes exhibited H3K4me3 levels that correlated with the severity of intestinal inflammation in IBD, despite lacking significant differential expression. Collectively, these data uncover a previously unrecognized epigenetic profile of IBD that can be primed by commensal microbes and indicate sensitive targets in the epithelium that may underlie how microbiota predispose to subsequent intestinal inflammation and disease.

Conflict of interest: The authors have declared that no conflict of interest exists.

Submitted: May 15, 2018 Accepted: August 7, 2018 Published: September 20, 2018

Reference information: JCI Insight. 2018;3(18):e122104. https://doi.org/10.1172/jici. insight.122104.

\section{Introduction}

Inflammatory bowel disease (IBD) affects over 1.4 million people in the United States and represents a significant public health and economic challenge (1-3). In pediatric patient populations, specifically, the incidence and prevalence of IBD continues to rise (4). It is clear that the pathogenesis of IBD is multifactorial, involving complex interactions between the environment and genetics of the host (5-8). While abnormal intestinal immune responses are a defining feature of IBD, the mechanisms triggering IBD in genetically susceptible individuals are poorly understood. Current therapeutics focus on controlling excessive inflammation, rather than upstream pathways that predispose to altered intestinal immune response. These treatment options can be effective in ameliorating symptoms in many patients, yet treatment failure is common, side effects of these therapies can be significant, and disease complications occur despite appropriate therapy $(9,10)$. Therefore, improved understanding of the molecular and cellular mechanisms 
mediating how environmental cues are integrated by intestinal cells to trigger IBD will enable development of new personalized approaches to treat the disease and predict response to therapy.

Commensal bacterial populations in the intestinal environment clearly associate with altered gene expression and the severity of Crohn's disease (CD), a prevalent form of IBD (7-9, 11-21). Thus, deciphering how the microbiota induces and sustains altered gene regulation in the intestine may reveal new targets for combatting disease. Intestinal epithelial cells (IECs) reside at the direct interface between the mammalian host and intestinal microbiota. These cells play a critical role in responding to commensal bacterial-derived signals to dynamically maintain barrier function through several adaptations, such as tight junction proteins, antimicrobial peptides, pattern recognition receptors, and immune modulatory cytokines (7, 22-30). However, the molecular pathways underlying how IECs integrate diverse microbiota-derived cues from the intestinal environment to regulate host responses remain poorly understood.

Epigenetic modifications can regulate gene expression in response to environmental cues, without altering the genetic sequence (31). Epigenetic modifications of the DNA and histones modify chromatin structure and transcription factor binding in a manner that enables transcriptional priming, activation, or repression. Therefore, epigenetics represent an important, potentially reversible, mechanism that may link genetic predisposition and environmental triggers in the pathogenesis of IBD (8). Sera and affected mucosa of $\mathrm{CD}$ patients exhibit changes in DNA methylation and global histone acetylation, although the dependence of these epigenetic modifications on environmental signals is not clear (32-37). In recent work, we identified an epigenetic regulator in IECs that integrates commensal bacterial-derived signals to regulate intestinal homeostasis and susceptibility to intestinal inflammation in the presence of commensal bacteria $(38,39)$. Thus, microbiota-induced alterations in epigenetic regulation may be a critical initiating trigger in the development and progression of IBD.

Here, we identify an ileal epithelial cell histone methylation signature in newly diagnosed, treatment-naive pediatric CD patients that has not been previously linked with IBD. Further, a significant proportion of the genes that make up this signature exhibit similar histone methylation patterns in the ilea of mice due to exposure to commensal bacteria, suggesting that epigenetic regulation of these targets may reflect a critical mechanism for how microbiota predispose to intestinal inflammation. Remarkably, the profile of several microbiota-sensitive targets correlated with severity of intestinal inflammation and disease in pediatric CD patients, revealing a clinically relevant epigenetic signature in the intestinal epithelium.

\section{Results}

Histone methylation profile of epithelium differentiates pediatric CD patients. Previous studies examining DNA methylation indicate that this modification is altered in serum and intestine of pediatric IBD patients (33-35, 37, 40-42); however, evaluation of links between epigenetics in IBD and regulation by the microbiota as well as extension to histone modifications has been limited. Thus, to undertake the initial investigation into whether histone modifications characterize the epithelial landscape in CD, ileal IECs from CD patients at disease onset and healthy controls were processed for ChIP sequencing (ChIP-seq) of the activating histone H3-lysine 4 trimethylation (H3K4me3) modification. IECs were compared between controls and CD patients, representing adolescents with an equal sex distribution (Table 1). Substantial differences in $\mathrm{H} 3 \mathrm{~K} 4 \mathrm{me} 3$ enrichment were identified within many genes in the ilea of pediatric $\mathrm{CD}$ patients, as indicated at representative genes in control and CD patients (Figure 1, A and B). Interestingly, examination of $\mathrm{H} 3 \mathrm{~K} 4 \mathrm{me} 3 \mathrm{ChIP}$-seq data across all 8 patient samples per group highlighted 1,066 shared sites (1,038 genes), with significantly increased H3K4me3 in CD patients, and 539 sites (548 genes) characterized by significantly reduced $\mathrm{H} 3 \mathrm{~K} 4 \mathrm{me} 3$ in $\mathrm{CD}$ patients compared with controls (Figure 1C). This H3K4me3 signature was significantly different across CD patients compared with controls, despite variability in clinical and histologic findings (Figure 1D and Table 1). While histologic features tracked with $\mathrm{H} 3 \mathrm{~K} 4 \mathrm{me} 3$ variability in some of these targets (Supplemental Figure 1; supplemental material available online with this article; https://doi.org/10.1172/jci.insight.122104DS1), it did not account for all variance observed within the CD cohort (Figure 1D). Further examination revealed that CD-specific sites of differential $\mathrm{H} 3 \mathrm{~K} 4 \mathrm{me} 3$ occurred predominately in promoters, although a significant proportion were also identified within genomic introns (Figure 1E).

Of the CD-specific epigenetically modified genes, 63 have at least 1 reported association with IBD based on the DisGeNET database (Supplemental Table 1) and 14 genes have been linked to CD-associated risk loci (43) (Supplemental Table 2). However, the majority of targets have not yet been examined in relation to IBD 
Table 1. Characteristics of pediatric patients (newly diagnosed CD and controls)

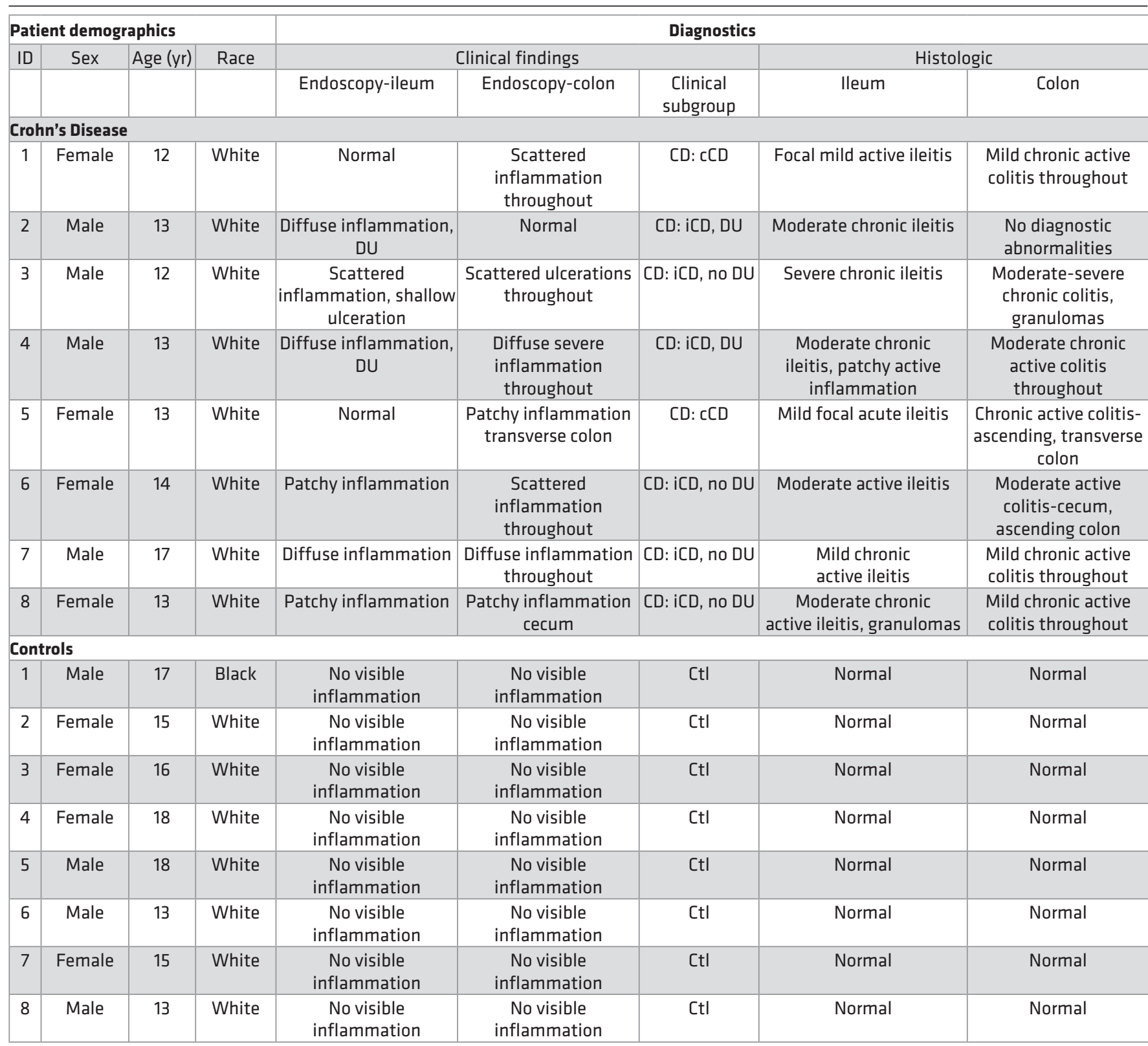

Crohn's disease (CD) subgroups are defined by clinically affected (ileal CD [iCD] with or without deep ulcers [DU]) and unaffected (colon-only CD [CCD]) ilea, in comparison with non-IBD controls (CtI).

pathogenesis and prognosis. Clustered pathway analyses of genes that were characterized by increased H3K4me3 signatures in $\mathrm{CD}$ strongly enriched for broad immune processes, including genes involved in epithelial response to bacterial factors and cytokine signaling (i.e., ICAM-1, PTGS-2, IL7) (Figure 2, A and B). Interestingly, genes represented by sites of decreased $\mathrm{H} 3 \mathrm{~K} 4 \mathrm{me} 3$ in $\mathrm{CD}$ predominantly regulate intestinal epithelial ion absorption as well as lipid and protein metabolism (Figure 2, C and D). Collectively, H3K4me3 status in IECs defined previously unrecognized genes and pathways that may be epigenetically regulated in treatment-naive pediatric IBD patients and could therefore represent novel targets for diagnostic evaluation and therapeutic intervention.

Microbiota regulate histone methylation of a unique subset of CD targets. Altered dynamics between IECs and the intestinal microbiota have been strongly linked to $C D$; however, whether epigenetic modifications in intestinal cells reflect integration of cues from microbiota-derived environmental signals is not known. 
A

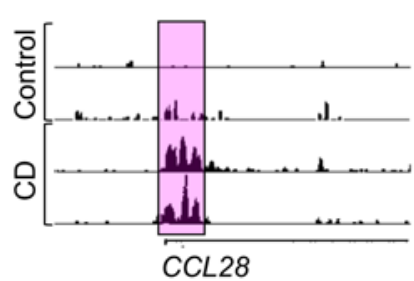

C

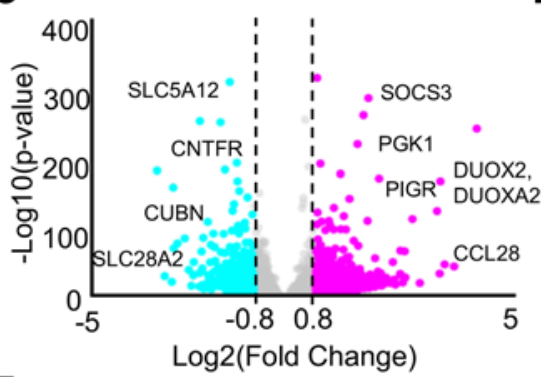

E

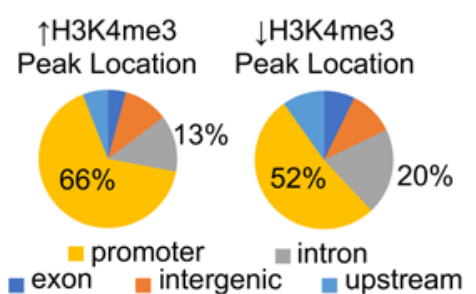

B

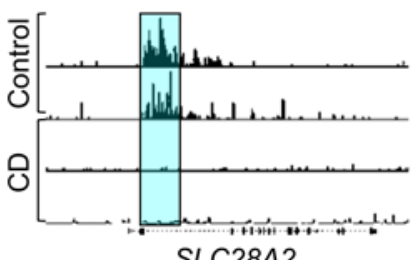

SLC28A2

D

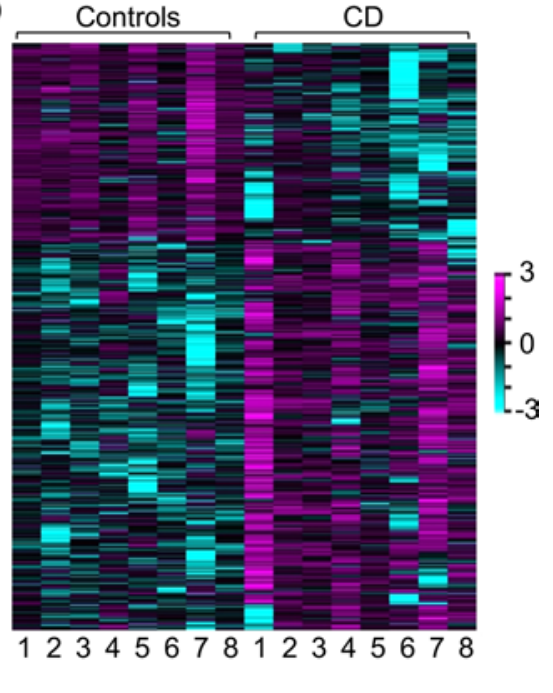

Figure 1. Histone methylation signature of ileal epithelium defines treatment-naive pediatric Crohn's disease patients. (A and B) Representative tracks from individual samples of differentially enriched peaks for genes with (A) increased H3K4me3 sites in Crohn's disease (CD) versus controls and (B) decreased H3K4me3 sites in CD. (C) Volcano plot representation of genes with differential H3K4me3 peaks $(P<0.05, n=8$ per group). (D) Heatmap of H3K4me3 enrichment across all samples for genes in C. (E) Genomic locations of sites with significantly increased or decreased H3K4me3 peaks in CD.

Given that the absence and presence of microbiota cannot be manipulated in patients, animal models need to be employed to identify whether IECs exhibit an epigenetic signature that is sensitive to the presence of intestinal microbiota. Therefore, to test whether commensal bacteria that reside in the intestine regulate $\mathrm{H} 3 \mathrm{~K} 4 \mathrm{me} 3$ in the distal small intestine, $\mathrm{H} 3 \mathrm{~K} 4 \mathrm{me} 3$ signatures in ileal epithelial cells of conventionally housed (CNV-housed) barrier mice were compared with cells harvested from germ-free (GF) mice that lack commensal microbes. These global analyses demonstrated that the microbiota did, in fact, induce significant alterations in H3K4me3 in IECs (Figure 3A). Notably microbiota-sensitive epigenetic targets included, but were not limited to, genes that have been previously characterized in epithelial antimicrobial responses, such as REG3G and NOS2 (44).

Similar to the distribution observed in IECs from CD patients (Figure 1E), microbiota-sensitive H3K4me3 sites were most commonly located within genomic promoters and introns (Figure 3B). Further, approximately 400 genes that exhibited differential $\mathrm{H} 3 \mathrm{~K} 4 \mathrm{me} 3$ in response to commensal microbes were regulated similarly at the epigenetic level in IECs of pediatric CD patients (Figure 3, C-H) in a manner more significant than by chance (increased H3K4me3, $P=3.8 \mathrm{e}-6$; decreased H3K4me3, $P=1.9 \mathrm{e}-16$ ). Of these overlapping targets, 327 genes exhibited significantly enriched H3K4me3 levels in IECs in CD as well as in response to microbes (Figure 3, C-E). Interestingly, DUOX2, an oxidase whose expression highly correlates with microbial responses in $\mathrm{CD}(45)$, demonstrated marked $\mathrm{H} 3 \mathrm{~K} 4 \mathrm{me} 3$ enrichment in both $\mathrm{CD}$ and in response to commensal bacteria (Figure 3D). Conversely, 97 genes that demonstrated loss of $\mathrm{H} 3 \mathrm{~K} 4 \mathrm{me} 3$ enrichment in IECs from CD patients relative to controls also exhibited decreased $\mathrm{H} 3 \mathrm{~K} 4 \mathrm{me} 3$ in IECs harvested from CNV mice relative to GF mice (Figure 3, F-H). The significance of most of these $\mathrm{CD}$-associated genes characterized by microbiota-sensitive decreased $\mathrm{H} 3 \mathrm{~K} 4 \mathrm{me} 3$, such as CUBN, THNSL2, and PCK1, has not yet been evaluated in IBD (Figure 3G).

Epigenetic signatures reveal microbiota-sensitive networks in IBD. To determine whether microbiota-sensitive epigenetic targets in pediatric CD (Figure 3, C and F) form significant functional relationships, clustered 
A

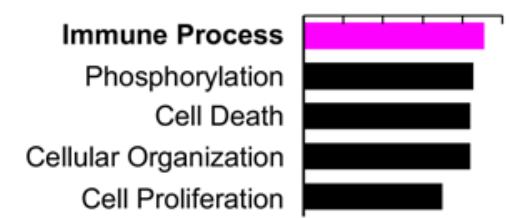

C

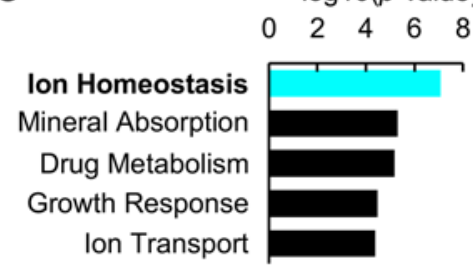

B

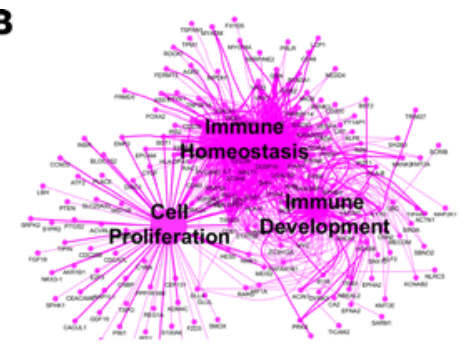

D

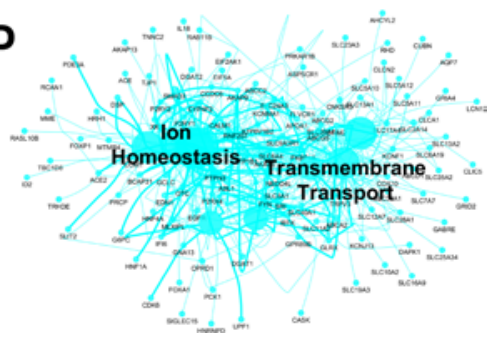

Figure 2. Histone methylation signature highlights epigenetically altered pathways in Crohn's disease patients. Clustered pathway analyses for genes with (A) increased or (C) decreased H3K4me3 in CD. Network connectivity of the highest ranking gene set exhibiting (B) increased H3K4me3 or (D) decreased H3K4me3 in CD.

pathway analyses were performed for this refined subset of genes. Dysregulated pathways that were identified based on global epigenetic profiles in $\mathrm{CD}$ (Figure 2), such as regulation of the immune response, ion transport, and metabolic pathways, were highly enriched specifically within this microbiota subset (Figure 4, A and B), supporting that the microbiota is a critical regulator of functional epigenetically marked pathways in $\mathrm{CD}$. In fact, targets in IECs of CD patients that were not responsive to the presence of microbiota (Figure 3, C and F) only exhibited enrichment for 76 pathways, whereas the smaller core set of overlapping microbiota-sensitive targets (Figure 3, C [327 genes] and F [97 genes]) enriched for 287 different pathways, highlighting high physiological potential for these microbiota targets in IBD.

Microbiota-sensitive genes that also demonstrate increased $\mathrm{H} 3 \mathrm{~K} 4 \mathrm{me} 3$ in IECs from $\mathrm{CD}$ patients were highly enriched in pathways involving production of reactive oxygen species (DUOX2, RAC1/2; $P=4.2 \mathrm{e}-6)$ and nitric oxide biosynthesis ( $A S S 1, N O S 2 ; P=1.0 \mathrm{e}-4)$ (Figure $4 \mathrm{~A})$. Genes regulating the innate mucosal barrier (MUC2, TGF- $\beta, I L 16, I L 2 R B$ ) were also enriched in this subset. Notably, targets less commonly evaluated in relation to IBD pathogenesis were also revealed. For instance, ephrin signaling that has been linked to immune signaling and epithelial homeostasis (46-49) was highlighted in this core subset (Figure 4A). Microbiota-sensitive targets characterized by loss of the H3K4me3 modification largely represent regulators of nutrients and metabolites in the intestine. Genes driving enrichment of these pathways included regulators of protein digestion and absorption (XPNPEP2, SLC7A7; $P=5.0 \mathrm{e}-4)$ and bile transport (SLC10A2, ABCC2; $P=5.5 \mathrm{e}-4$ ) (Figure 4B). Epigenetic analyses also indicated that expression of multiple genes that mediate mineral absorption exhibited microbiota- and CD-specific decreases in $\mathrm{H} 3 \mathrm{~K} 4 \mathrm{me} 3$ in IECs (Figure 4B). Thus, intestinal absorption deficiencies that occur in IBD may reflect an epithelial-intrinsic component in addition to alterations that occur subsequent to local damage and inflammation (50).

H3K4me3 represents an epigenetic mark of open or transcriptionally active chromatin where transcription factors bind DNA and alter transcriptional output. Therefore, to test whether microbiota-sensitive H3K4me3 sites in IECs of CD patients (Figure 3, C and F) share characteristic binding sites for known transcription factors, extensive global motif characterization was conducted. Examination of microbiota-sensitive sites with differential H3K4me3 enrichment in $\mathrm{CD}$ identified AP2 and NF- $\mathrm{BB}$ consensus sequences as enriched in promoter regions with $\mathrm{CD}$-specific $\mathrm{H} 3 \mathrm{~K} 4 \mathrm{me} 3$ induction (Figure 4, C and D). MEF2A and multiple FOXO transcription factor-binding sites were within promoters characterized by decreased $\mathrm{H} 3 \mathrm{~K} 4 \mathrm{me} 3$ (Figure 4, E and F). Collectively, these findings suggest that transcriptional regulation by factors such as AP2 and FOXO may be dysregulated in ileal IECs of IBD patients.

Microbiota-induced epigenetic targets associate with disease state and inflammation in $C D$. To investigate whether microbiota-sensitive epigenetically modified targets in $\mathrm{CD}$ track with transcriptional expression, RNA sequencing (RNA-seq) was performed on the same patient IECs that were analyzed for H3K4me3 by 
A

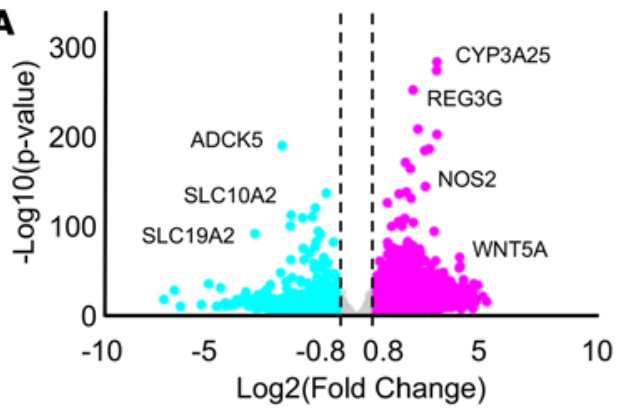

C

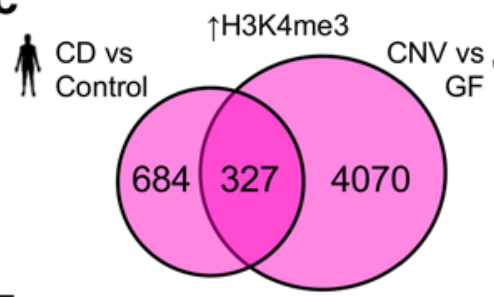

E

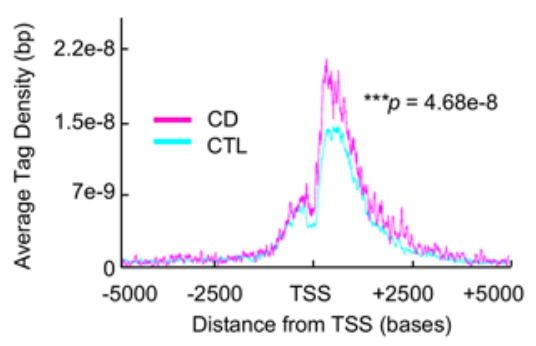

$\mathbf{F}$

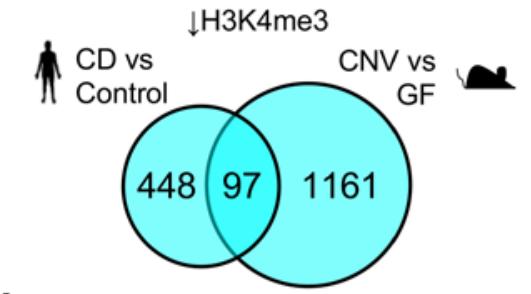

H

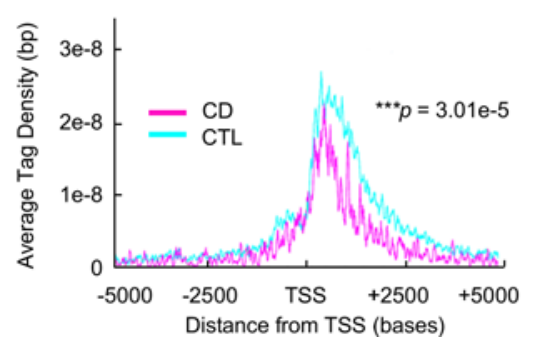

B

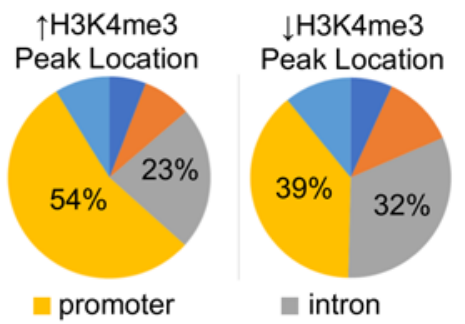

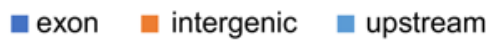

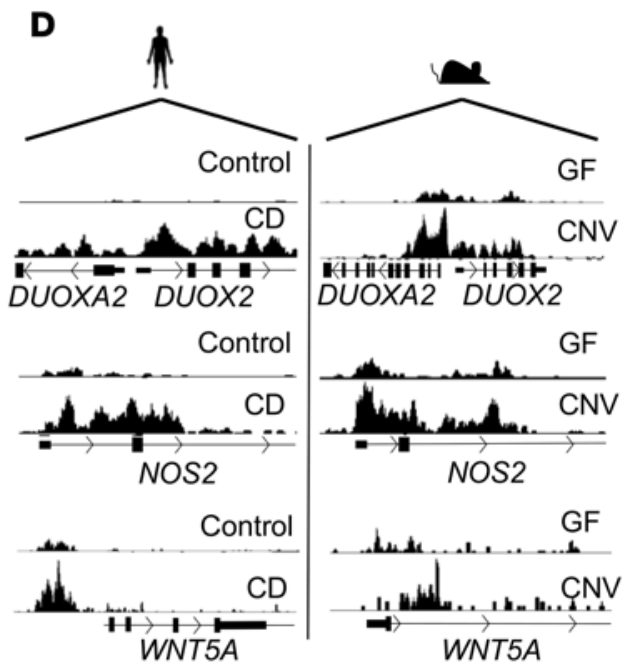

G

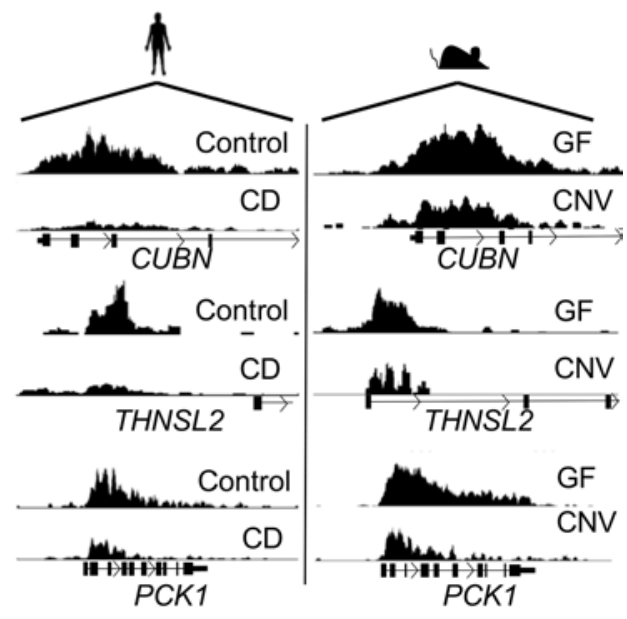

Figure 3. Microbiota regulate histone methylation of a unique subset of epithelial Crohn's disease targets. (A) Volcano plot representation of genes with differential H3K4me3 peaks in IECs from microbiota-replete conventionally housed (CNV) mice relative to germ-free (GF) mice ( $P<0.05, n=3$ per group). (B) Genomic locations of sites with significantly increased or decreased H3K4me3 peaks in IECs due to the presence of microbiota. (C) Venn diagram showing overlap of genes with increased $\mathrm{H} 3 \mathrm{~K} 4 \mathrm{me} 3$ in $\mathrm{CD}$ due to microbiota exposure ( $P=3.8 \mathrm{e}-06$ by hypergeometric test). (D) Representative sample tracks of peaks with increased H3K4me3 in IECs of CD patients and CNV mice. (E) Average H3K4me3 density for microbiota-sensitive genes in $C D$ from C. Wilcoxon rank-sum test was used to determine the $P$ value. (F) Venn diagram showing overlap of genes with decreased H3K4me3 in CD due to microbiota exposure ( $P=1.9 \mathrm{e}-16$ by hypergeometric test). (C) Representative sample tracks of peaks with decreased H3K4me3 in IECs of CD patients and CNV mice. (H) Average H3K4me3 density for microbiota-sensitive genes in $C D$ from $\mathbf{F}$. Wilcoxon rank-sum test was used to determine $P$ value.

ChIP-seq. Analyses of normalized mRNA expression across patients highlighted several microbiota-sensitive epigenetic targets that exhibited significantly altered expression in $\mathrm{CD}$ patients (Figure $5, \mathrm{~A}$ and $\mathrm{B}$, left). These genes demonstrated significant differential expression $(P \leq 0.05$, fold change $\geq 2)$ that strongly paralleled their $\mathrm{H} 3 \mathrm{~K} 4 \mathrm{me} 3$ epigenetic signature in CD patients (Supplemental Table 3). To address potential concerns about statistical power regarding RNA-seq data in this patient cohort, we expanded evaluation of microbiota-sensitive epigenetic targets to ileal biopsies from the larger pediatric RISK stratification cohort, which includes 179 pediatric CD patients and 38 control patients. Notably, the majority of genes identified in our initial analyses also exhibited marked differences in expression across this larger cohort of pediatric $\mathrm{CD}$ patients. Further, these targets also demonstrated a striking gradient in expression across disease subtypes in the RISK cohort, from no inflammation, to no macroscopic ileal inflammation (colon-only $\mathrm{CD}$ ), to active ileal inflammation (ileal CD) (Figure 5, A and B, right). A similar trend in expression was 
A

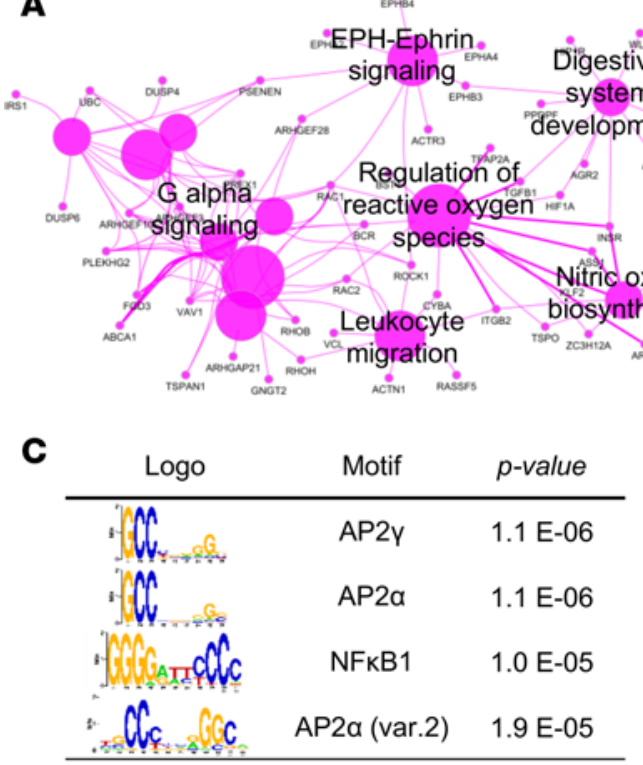

$\mathbf{E}$

\begin{tabular}{ccc} 
Logo & Motif & $p$-value \\
\hline MEF2A & $5.0 \mathrm{E}-06$ \\
FOXO1 & $6.0 \mathrm{E}-04$ \\
& SOX5 & $5.0 \mathrm{E}-06$ \\
\end{tabular}

B
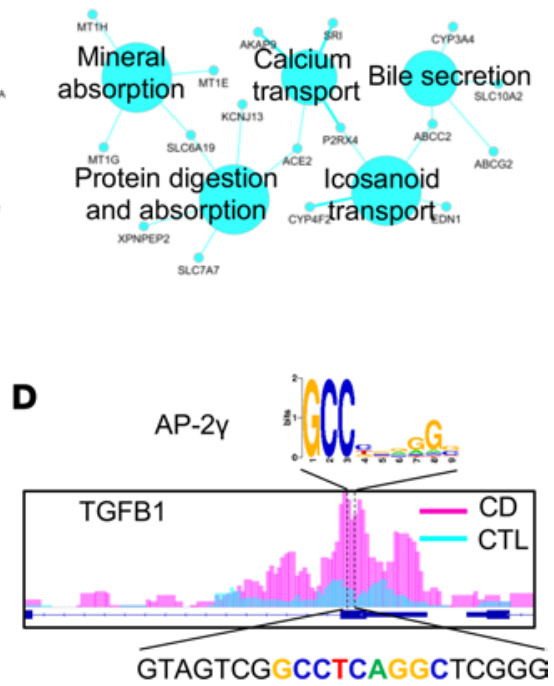

$\mathbf{F}$
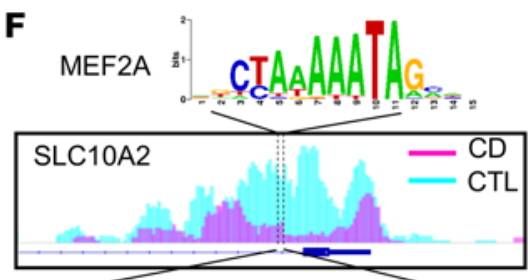

CTGTGAGCTAAAAACAGTAAGTC

Figure 4. Epigenetic signatures reveal microbiota-sensitive regulatory networks in IBD. (A and B) Pathway analyses of microbiota-sensitive genes with (A) increased or (B) decreased H3K4me3 in Crohn's disease (CD) versus controls (CTL). (C-F) Binding sites based on enriched motifs within 150 bp of H3K4me3 peaks in microbiota-sensitive genes in CD that are (C) increased and (E) decreased in CD intestinal epithelial cells. ( $D$ and $\mathbf{F}$ ) Representative motifs in target genes for (D) AP-2 and (F) MEF2A.

also observed for several of these targets in relation to clinical $C D$ subgroups and corresponding histologic severity in the smaller pediatric cohort (Supplemental Table 4). Thus, the level of expression of these relatively few microbiota-sensitive targets in IECs seems to distinguish ileal-defined CD subtypes.

In order to further examine the relationship between expression of these key targets and intestinal inflammation in IBD patients, their expression was examined relative to the inflammatory marker S100 calcium-binding protein A8 (S100A8) that correlates with CD severity (51). Ileal IEC expression of the majority of these genes (69\%) exhibited a significant linear correlation with level of $S 100 A 8$ expression. Expression of representative $\mathrm{CD}$ enriched candidate genes, such as DUOXA2, correlated strongly with epithelial expression of the IBD inflammatory biomarker (Figure 5C). Further, this positive relationship between microbiota-sensitive epigenetic targets was shared when expression was compared with $5100 \mathrm{~A} 8$ across the larger RISK cohort (Figure 5D). Likewise, epithelial expression of downregulated genes, such as CUBN, negatively correlated with $S 100 \mathrm{~A} 8$ levels (Figure 5C), and this relationship was similarly conserved when expanded to compare the full RISK cohort (Figure 5D).

Given that these microbiota-sensitive genes were characterized with significant differences in histone methylation, association between this epigenetic signature and disease was further evaluated. Principle component analysis of $\mathrm{H} 3 \mathrm{~K} 4 \mathrm{Me} 3 \mathrm{ChIP}$-seq data demonstrated more distinct separation between control and $\mathrm{CD}$ cohorts relative to the separation observed with RNA-seq data for these targets (Figure 6A). Therefore, to test the relationship between histone methylation at microbiota-sensitive targets and inflammation, correlation between $S 100 A 8$ and H3K4me3 levels was examined. Several genes exhibited significant correlation between levels of H3K4me3 and S100A8 when examining control and CD samples (Figure 6B). Interestingly, of the 
A

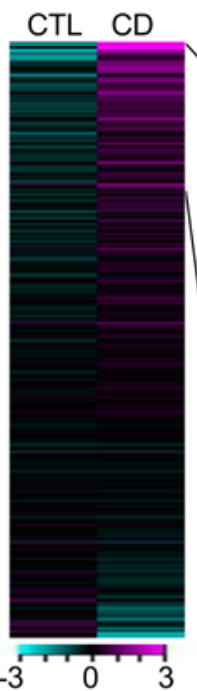

B
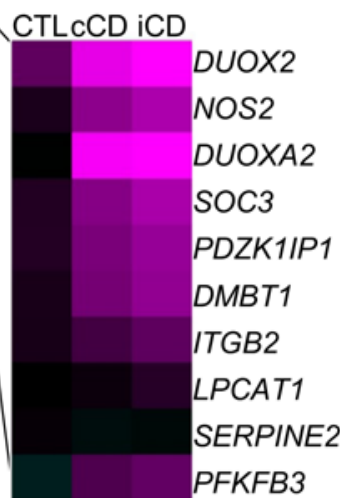

PFKFB3
C

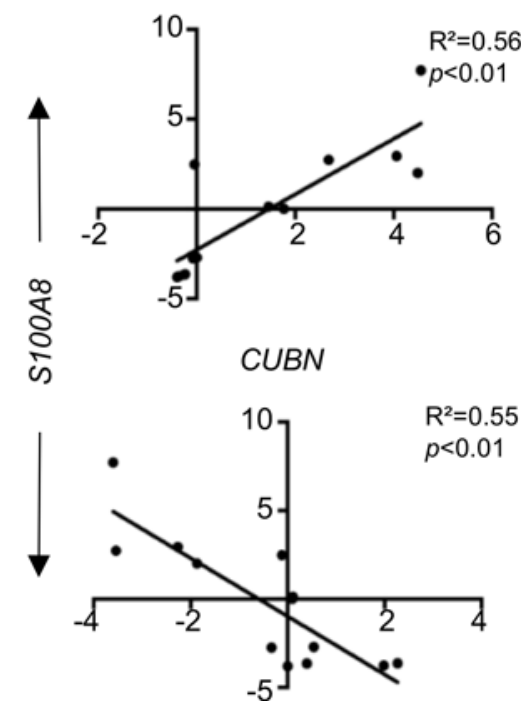

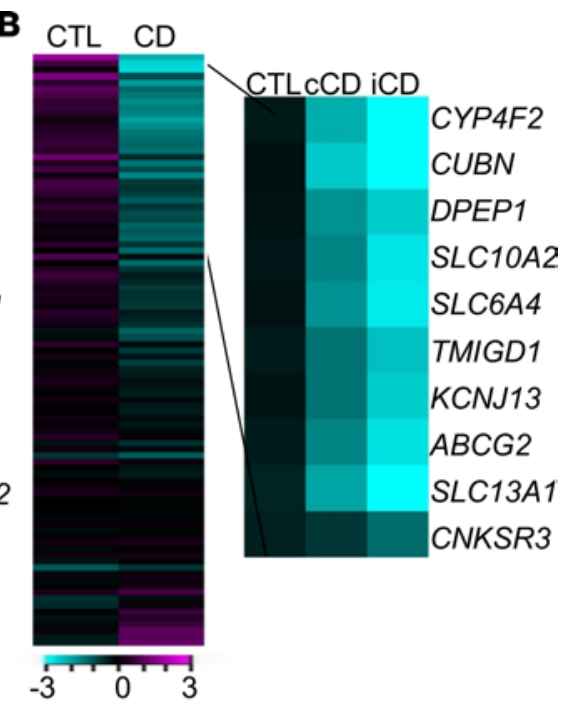

D
Figure 5. Microbiota-sensitive epigenetic targets are associated with disease state and inflammation in Crohn's disease. ( $A$ and B) Heatmap representation of differential gene expression by RNA-seq of microbiota-sensitive epigenetic targets in $C D$ that exhibited (A) increased or (B) decreased H3K4me3 (control [CTL, $n=7]$ and CD $[n=6]$ samples). Genes exhibiting largest differential expression with data from the IBD RISK cohort are shown on the right. (C) S100A8 expression versus expression of DUOXA2 and CUBN in the CCHMC IBD biorepository (CTL, $n=7 ; \mathrm{CD}, n=6$ ) and (D) RISK cohort (CTL, $n=$ 38; $C D, n=179$ ). Pearson correlation was used to determine $P$ values.
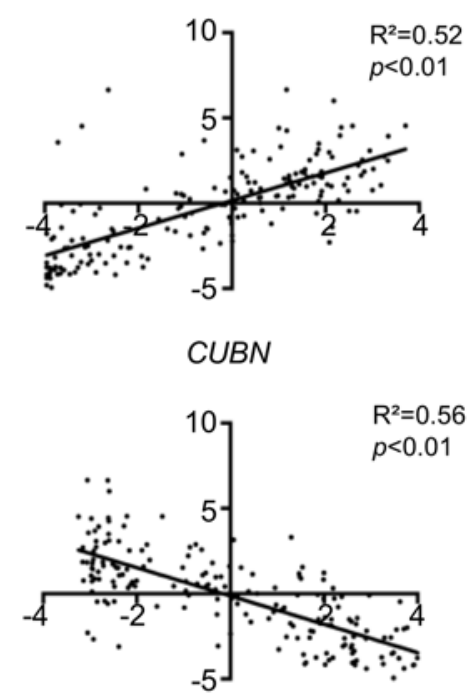

targets characterized as having a significant correlation between $\mathrm{H} 3 \mathrm{~K} 4 \mathrm{me} 3$ and S100A8, only 10\% exhibited significant differential expression in the same patients, whereas the majority (90\%) would not have been identified by expression analyses (Figure 6C). In fact, comparing this set of genes across the RISK cohort further confirmed that the majority of these genes ( 84 of 122; Supplemental Table 5) exhibited minimal differences in expression with disease. As an example of this from this epigenetically modified subset, histone methylation within IL7 and THNSL2 loci correlated with the level of intestinal inflammation in CD patients, despite a lack of significant change in expression (Figure 6D). Thus, microbiota-sensitive targets have been identified that exhibit epigenetic profiles that associate with $\mathrm{CD}$, and, importantly, traditional transcriptomics would be less likely to detect these potential candidates (Supplemental Table 6).

\section{Discussion}

By identifying sites of $\mathrm{H} 3 \mathrm{~K} 4 \mathrm{me} 3$ enrichment in patient IECs, this work reveals that the histone methylation profile of the ileal epithelium can effectively distinguish pediatric CD patients from patients that lack intestinal inflammation. Importantly, employing newly diagnosed, treatment-naive pediatric patients circumvented the common and significant challenge of distinguishing CD-associated alterations in the host from effects resulting from therapeutic exposure. Epigenetic dysregulation in IECs of CD patients is strongly enriched in genes that regulate cytokine signaling, metabolic homeostasis, and regulation of reactive oxygen species. Previous epigenetic studies in adult IBD have focused on DNA methylation and 
A

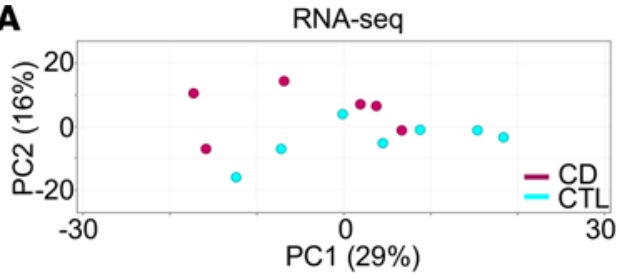

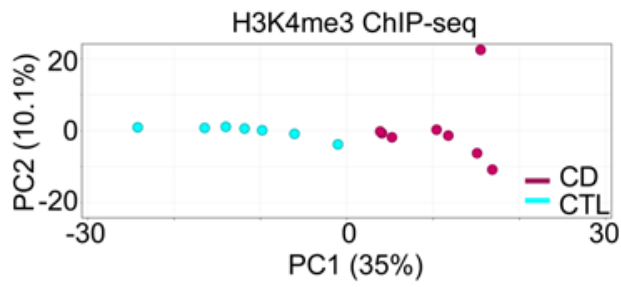

PC1 $(35 \%)$
B

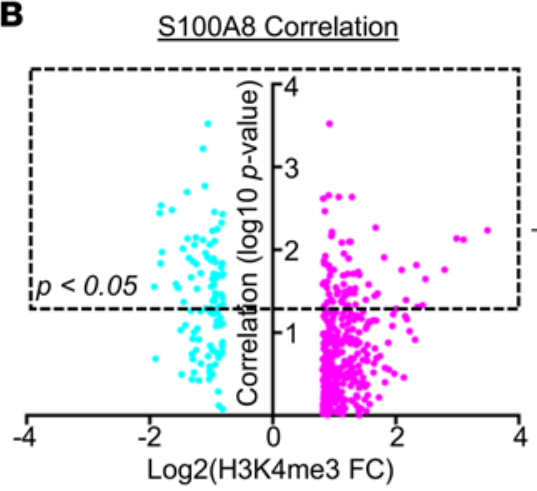

C

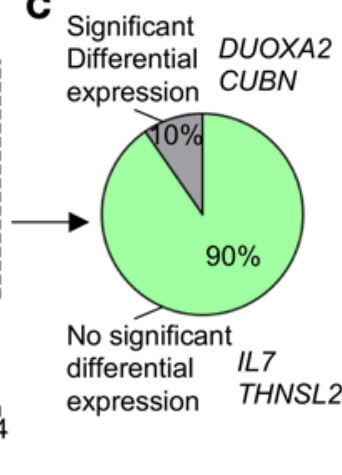

D

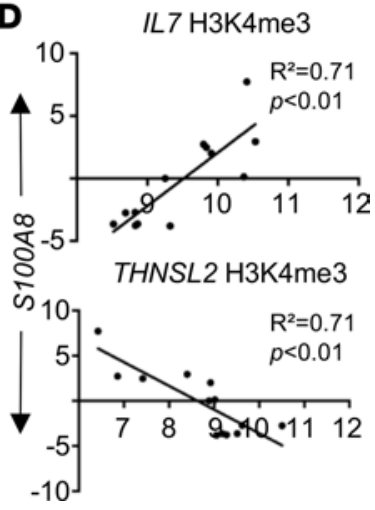

Figure 6. Histone methylation levels at microbiota-sensitive targets directly correlate with S100A8 in Crohn's disease. (A) PCA plots of RNA-seq and ChIP-seq data for microbiota-sensitive genes. (B) Pearson correlation $P$ value for the comparison of S100A8 expression and H3K4me3 levels of microbiota-sensitive genes. The box indicates a correlation with $P<0.05$. FC, fold change. (C) Pie chart indicating the percentage of targets in the box (B) with or without significant differential expression. (D) H3K4me3 level of IL7 and THNSL2 versus S100A8 expression.

identified different subsets of genes than those observed here for histone methylation (33-37, 40-42). Thus, chromatin alterations in histone methylation form a distinct epigenetic signature in IECs that differs in IBD patients compared with healthy controls at the time of their initial diagnosis. Despite relatively small patient numbers in this newly diagnosed, treatment-naive pediatric cohort, there was remarkable commonality in regions of altered histone methylation relative to controls. Given that samples used for ChIP and histologic analyses were distinct, it is not surprising that histologic interpretation did not always parallel the magnitude of epigenetic variability within this smaller cohort. Increased sample number and complimentary studies using inflamed and noninflamed mucosa from the same patient will enable future discovery of more subtle epigenetic differences and comprehensive histologic comparisons.

Beyond defining a histone methylation profile in the ilea of CD patients, this work goes further to predict which CD-specific epigenetic marks in the epithelium may be sensitive to the microbiota. Most striking is that the histone methylation signature itself often correlated with a molecular marker of inflammation in IBD. Many of these genes, in fact, do not exhibit significant differential expression and would, therefore, not be identified through transcriptomics to be clinically linked to IBD, highlighting that epigenetic analyses represent a complimentary approach for identification of epithelial molecular abnormalities. For example, $I L 7$, a gene that is characterized as epigenetically modified in CD epithelium, regulates mucosal lymphocytes (52) and is produced by epithelial cells in response to commensal microbes (53), suggesting that the threshold to induce IL7-dependent intestinal inflammation may be lower in CD patients or affected by other epigenetic modifications. As such, these types of targets may represent a group that is epigenetically primed by the microbiota during the onset of disease and could more likely exhibit transient alterations in expression or even enhanced response following exposure to additional disease-associated environmental triggers in the course of IBD.

Binding sites for AP2 and FOXO are enriched at microbiota-sensitive H3K4me3 loci in CD ileal epithelium, suggesting that altered regulation by these families of transcription factors may be particularly critical in early stages of IBD pathogenesis and may represent a potential target for therapy. Similarly, NF- $\kappa B$ motifs are also enriched at regions characterized by increased $\mathrm{H} 3 \mathrm{~K} 4 \mathrm{me} 3$, highlighting that the $\mathrm{CD}$ epigenetic profile identifies critical immunoregulatory regions in the genome that may be primed by the microbiota to drive enhanced inflammatory responses in reaction to subsequent environmental triggers. Progression of 
IBD has been linked to the composition of commensal bacteria, and, remarkably, we found that expression and/or H3K4me3 levels of specific microbiota-sensitive targets correlate with those in CD clinical subgroups and with S100A8, a marker of intestinal inflammation. Some of these genes, such as DUOX2 and DUOXA2, have been found to play a role in bacterial sensing and exhibit increased expression in the ilea of CD patients (45). In fact, DUOX2 drives ileitis in transgenic mice lacking glutathione peroxidase 1 and 2 (54). Many aspects of intestinal physiology and immune homeostasis are sensitive to the microbiota. Thus, the microbiota-sensitive alterations characterized using this murine GF comparison reflect a combination of both direct and indirect effects of intestinal colonization and may not fully recapitulate exposure to the $\mathrm{CD}$-associated microbiota compositions in humans. However, identification of microbiota-sensitive sites in these targets with $\mathrm{H} 3 \mathrm{~K} 4 \mathrm{me} 3$ alterations in $\mathrm{CD}$ highlights regions of epigenetic dysregulation that can now be examined in relation to microbiota composition.

Notably, this study highlights targets and pathways that are less commonly considered with regards to IBD pathogenesis. For instance, ephrin signaling genes, which have indeed been linked to immune signaling and epithelial homeostasis (46-49), are now shown to represent exciting targets that are epigenetically modified due to the intestinal microbiota. Thus, microbial-primed ephrin signaling in the intestine may be a critical, but yet unexplored, early trigger in IBD. In addition to genes characterized by elevation of the activating H3K4me3 mark, many genes were also identified with frequent reduction of this modification in the ileal epithelium of $\mathrm{CD}$ patients. Altered intestinal electrolyte transport has been described in the pathogenesis of IBD, particularly with regards to understanding disease-associated diarrhea (55). Sodium transport, in particular, appears to exhibit significant epigenetic dysregulation, and previous work has, in fact, suggested a potential role for intestinal sodium regulation in IBD (55). As an example, the SLC9A3R1 gene encoding a sodium-hydrogen regulator is characterized in $\mathrm{CD}$ with decreased $\mathrm{H} 3 \mathrm{~K} 4 \mathrm{me} 3$ enrichment and is downregulated in IBD (56). Epigenetic analyses also indicate that $C U B N$, a gene encoding a vitamin B12 receptor, displays a remarkably consistent decrease in histone methylation and expression in $\mathrm{CD}$. Interestingly, vitamin B12 deficiency is a common deficiency in IBD patients, and these patients may require vitamin B12 supplementation (57). Electrolyte and vitamin imbalances in IBD have been commonly attributed to intestinal damage caused by local inflammation $(50,58)$. However, our data suggest that the microbiota could trigger epigenetic alterations that reflect downregulation of epithelial absorption in the intestine. In fact, dysbiosis and sodium flux often do not normalize with otherwise successful anti-TNF therapy, even in asymptomatic CD patients $(59,60)$. Therefore, the microbiota may be a key factor that sustains altered ion and nutrient homeostasis in the intestine through epigenetic restructuring early in the course of IBD development.

Collectively, this microbiota-dependent $\mathrm{H} 3 \mathrm{~K} 4 \mathrm{me} 3$ signature may identify targets that are epigenetically modified due to microbiota exposure during the onset of disease. This work forms the critical entry point defining how microbiota-derived signals may be integrated by the host, emphasizing a fundamental role of epigenetics in priming epithelium for overt clinical disease with subsequent disease-associated environmental triggers. As progression of IBD can be tightly influenced by a patient's unique microbiota composition, identification of $\mathrm{H} 3 \mathrm{~K} 4 \mathrm{me} 3$ sites highlights regions of epigenetic dysregulation that can now be examined mechanistically in relation to other histone modifications as well as human microbiota using gnotobiotic colonization approaches. These studies will direct more specific approaches for individualized prevention and/or treatment of IBD based on modulation of epigenetically dysregulated pathways. In addition, assessing epithelial epigenetic targets in intestinal biopsies may provide a sensitive marker of intestinal inflammation and distinguish patients that could be more responsive to microbial-based therapies.

\section{Methods}

Patients. Children and adolescents undergoing colonoscopy were recruited prospectively for participation in the IBD biorepository protocol between 2015 and 2017 at CCHMC. Adolescents, between the ages of 12 and 18 years, with treatment-naive $C D$ were selected for this study, along with age- and sex-matched controls. Control patients presented due to intestinal symptoms, and colonoscopy was warranted as part of their evaluation. These patients were found to have no evidence of intestinal disease based on a combination of endoscopy, histology, lab testing, and imaging. Molecular analyses were performed on pooled biopsy samples collected together by endoscopy from sites in the ileum that exhibited representative gross pathology for the patient, ranging from no visible disease to severe inflammation, with or without ulceration. Collecting direct biopsies of ulcers was avoided. Histological analyses were performed using separate biopsies collected from multiple locations throughout both the small and large intestine. 
Animals. Mice were housed up to 4 per cage in a ventilated isolator cage system in a 12-hour-light/dark cycle, with free access to water and chow. GF mice were maintained in plastic isolator units in the CCHMC Gnotobiotic Mouse Facility, fed autoclaved feed and water, and routinely monitored to ensure the absence of microbial contamination. All experiments were performed with age- and sex-matched C57BL/6 mice.

IEC isolation. For isolation of IECs, samples were incubated with $1 \mathrm{mM}$ EDTA and $2 \mathrm{mM}$ DTT while shaking at $37^{\circ} \mathrm{C}$ at a $45^{\circ}$ angle for 10 minutes to obtain high epithelial purity with similarly small amounts of $\mathrm{CD} 45^{+}$cells $(\leq 5 \%)$ in both control and CD samples. Supernatant with IECs was pipetted into a new 50-ml tube and pelleted at $500 \mathrm{~g}$ for 5 minutes at $4^{\circ} \mathrm{C}$. A portion of IECs was processed for ChIP-seq, and $100 \mu 1$ RNAlater (Ambion) was added to the remainder for RNA-seq analyses. Mouse IECs were harvested from $10 \mathrm{~cm}$ of the distal small intestine. The intestine was opened longitudinally, washed, and incubated in buffer containing $1 \mathrm{mM}$ EDTA, 2 mM DTT, and 5\% FBS in PBS for 10 minutes, while shaking at $37^{\circ} \mathrm{C}$ at a $45^{\circ}$ angle to isolate IECs.

ChIP-seq. Fresh formaldehyde was added to each IEC sample for a final concentration of $1 \%$ and mixed end over end at room temperature for 10 minutes to crosslink proteins to DNA. Cells were lysed with a Triton X-100 and Igepal buffer (0.25\% Triton C-100, 0.5\% Igepal, 10\% glycerol, $1 \mathrm{mM}$ EDTA, $140 \mathrm{mM}$ $\mathrm{NaCl}$, and $50 \mathrm{mM} \mathrm{HEPES}$ ). Nuclei were then washed and chromatin sheared to 150 - to $500-\mathrm{bp}$ size in a $0.1 \%$ SDS in TE buffer using a S220 Covaris Sonicator. Immunoprecipitation was carried out with a SX-8G IP-STAR robot (Diagenode) and an antibody optimized for H3K4me3 as described previously $(61,62)$. Sequencing was performed using an Illumina HiSeq 2500. All samples exhibited high-quality sequencing, reads were mapped to human genome hg19 or mouse genome mm9 with Bowtie, and peaks were identified with MACS2 $(63,64)$. Gene orthology mappings are from Ensembl BioMart (65).

RNA-seq. RNA was isolated using the RNeasy Kit (Qiagen). Directional polyA RNA-seq was performed by the Genomics, Epigenomics, and Sequencing Core at the University of Cincinnati. NEBNext Poly(A) mRNA Magnetic Isolation Module (New England BioLabs) was used for polyA RNA purification with the Apollo 324 system (WaferGen) and PrepX PolyA script for automated polyA RNA isolation. To study differential gene expression, individually indexed and compatible libraries were proportionally pooled ( 25 million reads per sample in general) for clustering in the cBot system (Illumina). Libraries at the final concentration of $15 \mathrm{pM}$ were clustered onto a single-read flow cell using the Illumina NEBNext Ultra Directional RNA Library Prep Kit and sequenced to $50 \mathrm{bp}$ on the Illumina HiSeq system. Sequence reads were aligned to the $\mathrm{Hg} 19$ genome by using the Illumina sequence analysis pipeline created by the Laboratory for Statistical Genomics and Systems Biology at the University of Cincinnati. Sequence reads were aligned to the reference genome using the TopHat2 aligner, and reads aligning to each known transcript were counted using Bioconductor packages for next-generation sequencing data analysis $(66,67)$. Differential expression analysis was performed using the negative binomial statistical model of read counts as implemented in the edgeR Bioconductor package (68). Significantly differentially expressed genes $(P<$ 0.05 ; fold change $\geq 2$ ) were compared with previously published RNA-seq analyses from the Crohn's \& Colitis Foundation-sponsored multicenter RISK study that enrolled pediatric control patients $(n=38)$ and CD patients with and without endoscopic ileal inflammation $(n=179)(18)$.

Computational analyses. Diagnostic confirmation of whether a subject had CD or not (control) was conducted prior to analyses. Global sequencing data were analyzed with Biowardrobe (69), and differential ChIP-seq data were analyzed with replicates in THOR (70). Sample signal was normalized based on peaks at housekeeping genes (EMC7, GPI, PSMB4, RAB7A, VCP, CHMP2A, VPS29). Differential peaks between $\mathrm{CD}$ and control groups with all replicates were detected using a Hidden Markov Model approach. Housekeeping genes were individually inspected to ensure no differential enrichment was detected. Significant differential enrichment was defined as Benjamini-Hochberg-corrected $P<0.05$ and fold change $\geq 1.75$. For GF versus CNV analyses, to restrict to the most significant targets, a $\log _{10}(P$ value $) \leq 4.5$ was used. Pathway enrichment analyses with clustering was performed using ClueGo in Cytoscape with a Bonferroni-corrected $P<0.05$ (71). Functional groups were created with a $\kappa$ score greater than 0.4 . Unclustered pathway analysis using Toppgene was employed with a false discovery rate $<0.05$ (72). PscanChIP was used to identify transcription factor-binding site motifs within $150 \mathrm{bp}$ of the center of the differential peaks identified (73). JASPAR and TRANSFAC databases were used to rank based on $P$ value. The DisGeNET database $(5 / 2017)$ identified individual genes associated with IBD, a specificity score, and the number of NCBI citations. PCA plots were made in GeneSpring GS (Agilent) based on $\log _{2}$-transformed ChIP-seq and RNA-seq data baselined to median of all samples. RNA-seq and ChIP-seq data have been deposited in GEO and are accessible through GEO series accession numbers GSE117875 and GSE117889, respectively. 
Statistics. All inclusion/exclusion criteria were preestablished. THOR assigned $P$ values employing biological ChIP-seq replicates, and $P$ values were considered significant at $P<0.05$. Significance of overlap of genes between groups was calculated based on hypergeometric distribution, with 17,094 possible protein coding orthologs. For linear correlation analyses, normalized and transformed gene expression and methylation data were plotted against S100A8 expression for each patient sample. Pearson correlation was used to determine $\mathrm{r}^{2}$ coefficient and corresponding $P$ values. All data met the assumptions of the statistical tests used.

Study approval. Studies in humans were performed with approval by the CCHMC Institutional Review Board. Parents and adult patients, age 18 and older, provided consent, and all patients, age 11 and older, provided assent. All murine experiments were performed according to the animal experimental guidelines upon approval of the Institutional Animal Care and Use Committee at CCHMC.

\section{Author contributions}

TA, DK, and LAD designed the studies. DK, VW, SJ, JW, and JM carried out experiments. DK, MK, YH, $\mathrm{RK}$, and $\mathrm{AB}$ assisted with computational analyses. LAD, BJA, MCD, JSH, JFM, RNB, MCS, TDW, and SK provided patient samples and clinical expertise. NS, MJR, and $\mathrm{MH}$ provided critical reagents and tools. TA and DK analyzed the data and wrote the manuscript.

\section{Acknowledgments}

We thank the S. Way, D. Haslam, and J. Qualls labs (Infectious Disease, CCHMC) and the H. Deshmukh lab (Pulmonology, CCHMC) for useful discussions and members of the Alenghat lab for critical reading of the manuscript. The RISK committee provided patient samples and clinical expertise. We thank CCHMC Veterinary Services, the CCHMC DNA Sequencing Core, and the University of Cincinnati Genomics, Epigenomics, and Sequencing Core for services. We also thank RISK investigators for patient recruitment and the Crohn's \& Colitis Foundation for RISK support. This research is supported by the National Institutes of Health (DK093784, DK114123, and DK116868 to TA; DK098231 to LAD; and T32 DK007727 to DK), and a Crohn's \& Colitis Foundation/Janssen/AGA award to TA. TA holds a Career Award for Medical Scientists from the Burroughs Wellcome Fund and is a Pew Scholar in the Biomedical Sciences, supported by the Pew Charitable Trust. This project is supported in part by Public Health Service grant P30 DK078392 and the CCHMC Trustee Award and Procter Scholar's Program.

Address correspondence to: Theresa Alenghat, 3333 Burnet Ave., MLC 7038, Cincinnati, Ohio, USA. Phone: 513.803.7498; Email: theresa.alenghat@cchmc.org.

1. Benchimol EI, Fortinsky KJ, Gozdyra P, Van den Heuvel M, Van Limbergen J, Griffiths AM. Epidemiology of pediatric inflammatory bowel disease: a systematic review of international trends. Inflamm Bowel Dis. 2011;17(1):423-439.

2. Shivashankar R, Tremaine WJ, Harmsen WS, Loftus EV. Incidence and Prevalence of Crohn's Disease and Ulcerative Colitis in Olmsted County, Minnesota From 1970 Through 2010. Clin Gastroenterol Hepatol. 2017;15(6):857-863.

3. Loftus EV. Clinical epidemiology of inflammatory bowel disease: Incidence, prevalence, and environmental influences. Gastroenterology. 2004;126(6):1504-1517.

4. Benchimol EI, et al. Trends in epidemiology of pediatric inflammatory bowel disease in canada: distributed network analysis of multiple population-based provincial health administrative databases. Am J Gastroenterol. 2017;112(7):1120-1134.

5. Sartor RB, Wu GD. Roles for intestinal bacteria, viruses, and fungi in pathogenesis of inflammatory bowel diseases and therapeutic approaches. Gastroenterology. 2017;152(2):327-339.e4.

6. Rosen MJ, Dhawan A, Saeed SA. Inflammatory bowel disease in children and adolescents. JAMA Pediatr. 2015;169(11):1053-1060.

7. Pastorelli L, De Salvo C, Mercado JR, Vecchi M, Pizarro TT. Central role of the gut epithelial barrier in the pathogenesis of chronic intestinal inflammation: lessons learned from animal models and human genetics. Front Immunol. 2013;4:280.

8. Renz H, von Mutius E, Brandtzaeg P, Cookson WO, Autenrieth IB, Haller D. Gene-environment interactions in chronic inflammatory disease. Nat Immunol. 2011;12(4):273-277.

9. Kugathasan S, et al. Prediction of complicated disease course for children newly diagnosed with Crohn's disease: a multicentre inception cohort study. Lancet. 2017;389(10080):1710-1718.

10. Kopylov U, Seidman E. Predicting durable response or resistance to antitumor necrosis factor therapy in inflammatory bowel disease. Therap Adv Gastroenterol. 2016;9(4):513-526.

11. Baumgart DC, Carding SR. Inflammatory bowel disease: cause and immunobiology. Lancet. 2007;369(9573):1627-1640.

12. Blumberg R, Powrie F. Microbiota, disease, and back to health: a metastable journey. Sci Transl Med. 2012;4(137):137rv7.

13. Ley RE, Peterson DA, Gordon JI. Ecological and evolutionary forces shaping microbial diversity in the human intestine. Cell. 2006;124(4):837-848.

14. Macdonald TT, Monteleone G. Immunity, inflammation, and allergy in the gut. Science. 2005;307(5717):1920-1925. 
15. Sun L, Nava GM, Stappenbeck TS. Host genetic susceptibility, dysbiosis, and viral triggers in inflammatory bowel disease. Curr Opin Gastroenterol. 2011;27(4):321-327.

16. Tremaroli V, Bäckhed F. Functional interactions between the gut microbiota and host metabolism. Nature. 2012;489(7415):242-249.

17. Packey CD, Sartor RB. Interplay of commensal and pathogenic bacteria, genetic mutations, and immunoregulatory defects in the pathogenesis of inflammatory bowel diseases. J Intern Med. 2008;263(6):597-606.

18. Haberman Y, et al. Pediatric Crohn disease patients exhibit specific ileal transcriptome and microbiome signature. J Clin Invest. 2014;124(8):3617-3633.

19. Shih DQ, Targan SR. Insights into IBD pathogenesis. Curr Gastroenterol Rep. 2009;11(6):473-480.

20. Strober W, Fuss I, Mannon P. The fundamental basis of inflammatory bowel disease. J Clin Invest. 2007;117(3):514-521.

21. Packey CD, Sartor RB. Commensal bacteria, traditional and opportunistic pathogens, dysbiosis and bacterial killing in inflammatory bowel diseases. Curr Opin Infect Dis. 2009;22(3):292-301.

22. Peterson LW, Artis D. Intestinal epithelial cells: regulators of barrier function and immune homeostasis. Nat Rev Immunol. 2014;14(3):141-153.

23. Kaser A, Niederreiter L, Blumberg RS. Genetically determined epithelial dysfunction and its consequences for microflora-host interactions. Cell Mol Life Sci. 2011;68(22):3643-3649.

24. Lewis K, McKay DM. Metabolic stress evokes decreases in epithelial barrier function. Ann N Y Acad Sci. 2009;1165:327-337.

25. Dahan S, Roth-Walter F, Arnaboldi P, Agarwal S, Mayer L. Epithelia: lymphocyte interactions in the gut. Immunol Rev. 2007;215:243-253.

26. Iweala OI, Nagler CR. Immune privilege in the gut: the establishment and maintenance of non-responsiveness to dietary antigens and commensal flora. Immunol Rev. 2006;213:82-100.

27. Booth C, Potten CS. Gut instincts: thoughts on intestinal epithelial stem cells. J Clin Invest. 2000;105(11):1493-1499.

28. Artis D. Epithelial-cell recognition of commensal bacteria and maintenance of immune homeostasis in the gut. Nat Rev Immunol. 2008;8(6):411-420.

29. Fritz JH, Ferrero RL, Philpott DJ, Girardin SE. Nod-like proteins in immunity, inflammation and disease. Nat Immunol. 2006;7(12):1250-1257.

30. Campbell EL, Serhan CN, Colgan SP. Antimicrobial aspects of inflammatory resolution in the mucosa: a role for proresolving mediators. J Immunol. 2011;187(7):3475-3481.

31. Arrowsmith CH, Bountra C, Fish PV, Lee K, Schapira M. Epigenetic protein families: a new frontier for drug discovery. Nat Rev Drug Discov. 2012;11(5):384-400.

32. Tsaprouni LG, Ito K, Powell JJ, Adcock IM, Punchard N. Differential patterns of histone acetylation in inflammatory bowel diseases. J Inflamm (Lond). 2011;8(1):1.

33. Cooke J, et al. Mucosal genome-wide methylation changes in inflammatory bowel disease. Inflamm Bowel Dis. 2012;18(11):2128-2137.

34. Harris RA, et al. Colonic mucosal epigenome and microbiome development in children and adolescents. J Immunol Res. 2016;2016:9170162.

35. Ventham NT, et al. Integrative epigenome-wide analysis demonstrates that DNA methylation may mediate genetic risk in inflammatory bowel disease. Nat Commun. 2016;7:13507.

36. Lin Z, et al. Identification of disease-associated DNA methylation in intestinal tissues from patients with inflammatory bowel disease. Clin Genet. 2011;80(1):59-67.

37. McDermott E, et al. DNA methylation profiling in inflammatory bowel disease provides new insights into disease pathogenesis. J Crohns Colitis. 2016;10(1):77-86.

38. Alenghat T, et al. Histone deacetylase 3 coordinates commensal-bacteria-dependent intestinal homeostasis. Nature. 2013;504(7478):153-157.

39. Navabi N, et al. Epithelial histone deacetylase 3 instructs intestinal immunity by coordinating local lymphocyte activation. Cell Rep. 2017;19(6):1165-1175.

40. Howell KJ, et al. DNA methylation and transcription patterns in intestinal epithelial cells from pediatric patients with inflammatory bowel diseases differentiate disease subtypes and associate with outcome. Gastroenterology. 2018;154(3):585-598.

41. Karatzas PS, Mantzaris GJ, Safioleas M, Gazouli M. DNA methylation profile of genes involved in inflammation and autoimmunity in inflammatory bowel disease. Medicine (Baltimore). 2014;93(28):e309.

42. Bae JH, Park J, Yang KM, Kim TO, Yi JM, IBD Study Group of Korean Association for Study of Intestinal Diseases (KASID). Detection of DNA hypermethylation in sera of patients with Crohn's disease. Mol Med Rep. 2014;9(2):725-729.

43. Marigorta UM, et al. Transcriptional risk scores link GWAS to eQTLs and predict complications in Crohn's disease. Nat Genet. 2017;49(10):1517-1521.

44. Haber AL, et al. A single-cell survey of the small intestinal epithelium. Nature. 2017;551(7680):333-339.

45. Grasberger H, et al. Increased expression of DUOX2 is an epithelial response to mucosal dysbiosis required for immune homeostasis in mouse intestine. Gastroenterology. 2015;149(7):1849-1859.

46. Perez White BE, Getsios S. Eph receptor and ephrin function in breast, gut, and skin epithelia. Cell Adh Migr. 2014;8(4):327-338.

47. Muñoz JJ, Cejalvo T, Alonso-Colmenar LM, Alfaro D, Garcia-Ceca J, Zapata A. Eph/Ephrin-mediated interactions in the thymus. Neuroimmunomodulation. 2011;18(5):271-280.

48. Sharfe N, Nikolic M, Cimpeon L, Van De Kratts A, Freywald A, Roifman CM. EphA and ephrin-A proteins regulate integrin-mediated T lymphocyte interactions. Mol Immunol. 2008;45(5):1208-1220.

49. Hafner C, et al. Ephrin-B2 is differentially expressed in the intestinal epithelium in Crohn's disease and contributes to accelerated epithelial wound healing in vitro. World J Gastroenterol. 2005;11(26):4024-4031.

50. Barkas F, Liberopoulos E, Kei A, Elisaf M. Electrolyte and acid-base disorders in inflammatory bowel disease. Ann Gastroenterol. $2013 ; 26(1): 23-28$.

51. Leach ST, et al. Serum and mucosal S100 proteins, calprotectin (S100A8/S100A9) and S100A12, are elevated at diagnosis in children with inflammatory bowel disease. Scand J Gastroenterol. 2007;42(11):1321-1331.

52. Laky K, et al. Enterocyte expression of interleukin 7 induces development of gammadelta T cells and Peyer's patches. $J$ Exp 
Med. 2000;191(9):1569-1580.

53. Shalapour S, et al. Commensal microflora and interferon-gamma promote steady-state interleukin-7 production in vivo. Eur $J$ Immunol. 2010;40(9):2391-2400

54. Chu FF, et al. Deficiency in Duox2 activity alleviates ileitis in GPx1- and GPx2-knockout mice without affecting apoptosis incidence in the crypt epithelium. Redox Biol. 2017;11:144-156.

55. Priyamvada S, Gomes R, Gill RK, Saksena S, Alrefai WA, Dudeja PK. Mechanisms underlying dysregulation of electrolyte absorption in inflammatory bowel disease-associated diarrhea. Inflamm Bowel Dis. 2015;21(12):2926-2935.

56. Sullivan S, et al. Downregulation of sodium transporters and NHERF proteins in IBD patients and mouse colitis models: potential contributors to IBD-associated diarrhea. Inflamm Bowel Dis. 2009;15(2):261-274.

57. Pan Y, et al. Associations between folate and vitamin b12 levels and inflammatory bowel disease: a meta-analysis. Nutrients. 2017;9(4).

58. Weisshof R, Chermesh I. Micronutrient deficiencies in inflammatory bowel disease. Curr Opin Clin Nutr Metab Care. 2015;18(6):576-581.

59. Allan R, Steinberg DM, Dixon K, Cooke WT. Changes in the bidirectional sodium flux across the intestinal mucosa in Crohn's disease. Gut. 1975;16(3):201-204.

60. Lewis JD, et al. Inflammation, antibiotics, and diet as environmental stressors of the gut microbiome in pediatric Crohn's disease. Cell Host Microbe. 2015;18(4):489-500.

61. Barski A, et al. Rapid recall ability of memory T cells is encoded in their epigenome. Sci Rep. 2017;7:39785.

62. Cuddapah S, et al. Native chromatin preparation and Illumina/Solexa library construction. Cold Spring Harb Protoc. 2009;2009(6):pdb.prot5237.

63. Langmead B, Trapnell C, Pop M, Salzberg SL. Ultrafast and memory-efficient alignment of short DNA sequences to the human genome. Genome Biol. 2009;10(3):R25.

64. Zhang Y, et al. Model-based analysis of ChIP-Seq (MACS). Genome Biol. 2008;9(9):R137.

65. Kasprzyk A. BioMart: driving a paradigm change in biological data management. Database (Oxford). 2011;2011:bar049.

66. Kim D, Pertea G, Trapnell C, Pimentel H, Kelley R, Salzberg SL. TopHat2: accurate alignment of transcriptomes in the presence of insertions, deletions and gene fusions. Genome Biol. 2013;14(4):R36.

67. Huber W, et al. Orchestrating high-throughput genomic analysis with Bioconductor. Nat Methods. 2015;12(2):115-121.

68. Anders S, et al. Count-based differential expression analysis of RNA sequencing data using R and Bioconductor. Nat Protoc. 2013;8(9):1765-1786.

69. Kartashov AV, Barski A. BioWardrobe: an integrated platform for analysis of epigenomics and transcriptomics data. Genome Biol. 2015;16:158.

70. Allhoff M, Seré K, F Pires J, Zenke M, G Costa I. Differential peak calling of ChIP-seq signals with replicates with THOR. Nucleic Acids Res. 2016;44(20):e153.

71. Mlecnik B, Galon J, Bindea G. Comprehensive functional analysis of large lists of genes and proteins. J Proteomics. 2018;171:2-10.

72. Chen J, Bardes EE, Aronow BJ, Jegga AG. ToppGene Suite for gene list enrichment analysis and candidate gene prioritization. Nucleic Acids Res. 2009;37(Web Server issue):W305-W311.

73. Zambelli F, Pesole G, Pavesi G. PscanChIP: Finding over-represented transcription factor-binding site motifs and their correlations in sequences from ChIP-Seq experiments. Nucleic Acids Res. 2013;41(Web Server issue):W535-W543. 